\title{
Directional Charge-Carrier Transport in Oriented Benzodithiophene Covalent Organic Framework Thin Films
}

\author{
Dana D. Medina, ${ }^{* \dagger,}, \perp$ Michiel L. Petrus, ${ }^{\dagger, \perp}$ Askhat N. Jumabekov, ${ }^{\S, \perp}$ Johannes T. Margraf, ${ }^{\ddagger, \|}$
} Simon Weinberger, ${ }^{\dagger}$ Julian M. Rotter, ${ }^{\dagger}$ Timothy Clark, ${ }^{\ddagger 0}$ and Thomas Bein ${ }^{* \dagger}$

${ }^{\dagger}$ Department of Chemistry and Center for NanoScience (CeNS), University of Munich (LMU), Butenandtstrasse 5-13, 81377 Munich, Germany

${ }^{\ddagger}$ Friedrich-Alexander-University Erlangen-Nürnberg (FAU), Computer-Chemie-Centrum, Nägelsbachstraße 25, 91052 Erlangen, Germany

${ }^{\S}$ CSIRO Manufacturing, Clayton, Victoria 3168, Australia

"Quantum Theory Project, University of Florida, Gainesville, Florida 32611, United States

\section{Supporting Information}

\begin{abstract}
Charge-carrier transport in oriented COF thin films is an important factor for realizing COF-based optoelectronic devices. We describe how highly oriented electron-donating benzodithiophene BDT-COF thin films serve as a model system for a directed charge-transport study. Oriented BDT-COF films were deposited on different electrodes with excellent control over film roughness and topology, allowing for high-quality electrode-COF interfaces suitable for device fabrication. Holeonly devices were constructed to study the columnar hole mobility of the BDT-COF films. The transport measure-

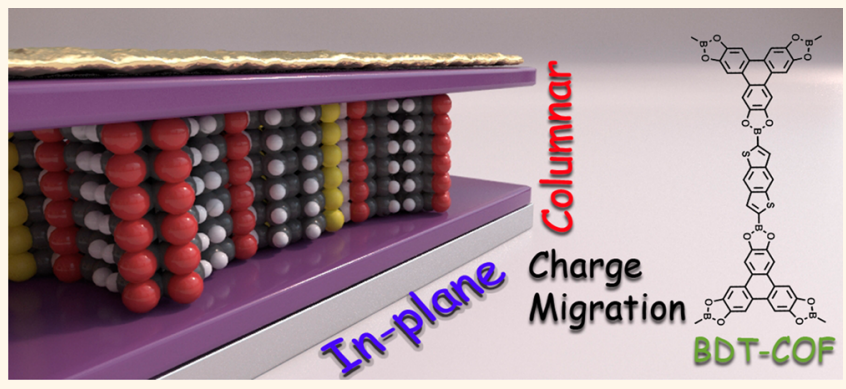
ments reveal a clear dependency of the measured hole mobilities on the BDT-COF film thickness, where thinner films showed about two orders of magnitude higher mobilities than thicker ones. Transport measurements under illumination yielded an order of magnitude higher mobility than in the dark. In-plane electrical conductivity values of up to $5 \times 10^{-7} \mathrm{~S}$ $\mathrm{cm}^{-1}$ were obtained for the oriented films. Impedance measurements of the hole-only devices provided further electrical description of the oriented BDT-COF films in terms of capacitance, recombination resistance, and dielectric constant. An exceptionally low dielectric constant value of approximately 1.7 was estimated for the BDT-COF films, a further indication of their highly porous nature. DFT and molecular-dynamics simulations were carried out to gain further insights into the relationships between the COF layer interactions, electronic structure, and the potential device performance.
\end{abstract}

KEYWORDS: covalent organic frameworks, thin films, charge transport, hole mobility, electrical conductivity, impedance spectroscopy

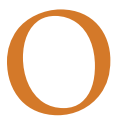

rganic semiconducting compounds are increasingly being developed as advanced materials for optoelectronic applications. Because versatile synthetic design strategies enable tunable mechanical and electronic material properties, organic semiconductors have been successfully integrated into a wide range of electronic devices. ${ }^{1,2}$ Many factors govern the performance of the organic layers in a device, where packing and long-range order are among the key features influencing the charge-carrier transport properties and therefore the final device performance. ${ }^{3,4}$ In this context, specific molecular alignments in a crystalline film and control over the film morphology can cause a significant improvement of the fundamental transport properties in organic electronics. ${ }^{2,5,6}$ Developing new platforms to control the structure-transport properties relationship is therefore of paramount importance. ${ }^{7}$
Layered alternating copolymers consisting of discrete subunits that self-assemble through dispersive forces allow for the formation of highly ordered, porous structures, so-called two-dimensional covalent organic frameworks (2D COFs). ${ }^{8-11}$ The slightly reversible nature of the covalent bond formation reactions connecting the $\mathrm{COF}$ subunits in combination with the mode of COF layer assembly enable, in principle, the formation of ordered molecular columns. Hence, the incorporation of electroactive subunits into a $2 \mathrm{D}$ COF backbone permits precise molecular positioning in a periodic

Received: November 15, 2016

Accepted: January 19, 2017

Published: January 19, 2017 
Scheme 1. (Left) Schematic Representation of BDT-COF. (Center and Right) COF Hole-Only Device Layout and the Corresponding Energy Diagram
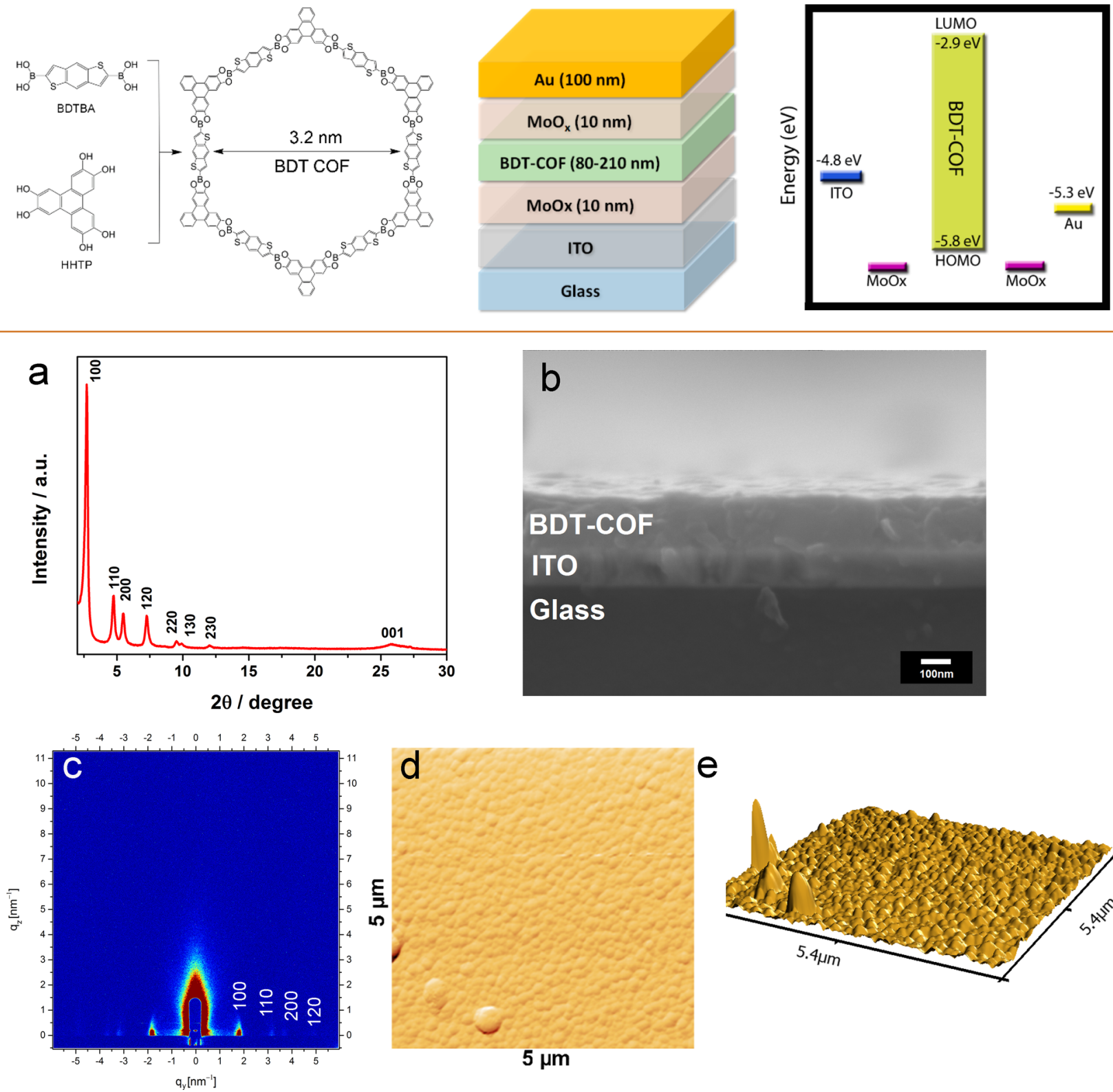

e

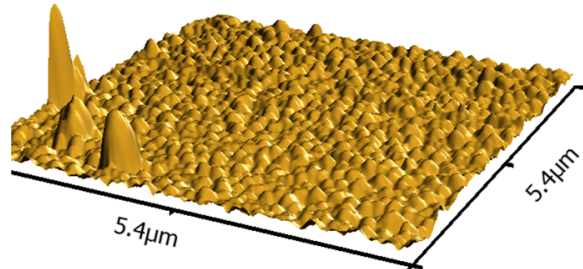

Figure 1. (Top) (a) Powder XRD pattern of BDT-COF isolated from the thin-film synthesis reaction mixture. (b) SEM micrograph of BDTCOF thin-film cross section with estimated film thickness of $200 \mathrm{~nm}$. (Bottom) (c) GISAXS pattern of BDT-COF thin film on ITO. (d) AFM image of $5 \times 5 \mu \mathrm{m}$ area and (e) 3D depiction of the film topography.

columnar manner. ${ }^{12-15}$ Therefore, charge migration in the columns of $2 \mathrm{D}$ COFs relies mainly on the interactions between adjacent COF layers. A detailed description of their transport properties is highly desirable in order to identify $2 \mathrm{D}$ COFs suitable for organic electronics. ${ }^{15-17}$ In this context, depositing 2D COFs on semiconducting surfaces is a first step toward COF-based device fabrication. ${ }^{18,19}$ Control over the orientation of the COF layers relative to the surface permits the investigation of the charge migration behavior in the plane and in the molecular columns of the COF. For these studies, thin COF films with a preferential layer orientation parallel to the surface are highly desirable.

Recently, we and others ${ }^{15,17-19}$ reported in a family of boronate ester electron-donating thiophene-containing
COFs. ${ }^{18,20-22}$ Light-induced charge separation and collection in a photovoltaic device was first demonstrated with a thienothiophene-based COF, TT-COF. ${ }^{20}$ Charge transfer from an electron-donating COF backbone to an acceptor phase was studied for oriented thin films of a benzodithiophene-based COF, BDT-COF. Dinca et al. reported the synthesis of BDT-COF analogues with heavy chalcogens and studied their electrical conductivity in the bulk. ${ }^{23}$

In this work, oriented BDT-COF thin films integrated into a device serve as model systems for studying directional chargecarrier transport and additional electrical properties. The hole mobility of the BDT-COF along the columnar stacks was studied in a diode configuration by constructing hole-only devices (HODs). We tested our premise that this model system 

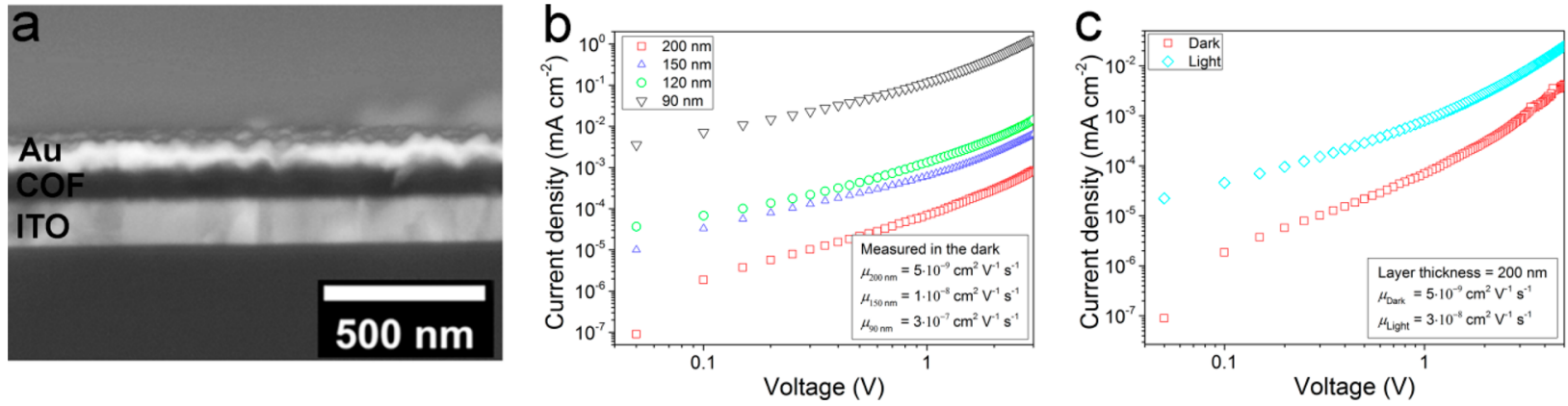

Figure 2. (a) SEM cross-section micrographs of $90 \mathrm{~nm}$ BDT-COF film HOD taken in backscattered electron mode. Current density as a function of voltage $(J-V)$ of hole-only devices containing BDT-COF layers of $(b)$ different film thicknesses and (c) under illumination and in the dark.

is a photoactive material by measuring columnar hole mobilities under illumination and in the dark. In-plane conductivity measurements of the BDT-COF along the COF layers provided a complementary electrical description of the system. Impedance measurements yielded additional electrical characteristics such as capacitance, recombination resistance and dielectric constant of the COF. Furthermore, theoretical investigations provide a link between the BDT-COF layer interactions and the charge transport along the BDT molecular stacks.

\section{RESULTS AND DISCUSSION}

Charge-carrier transport along the stacked columnar COF layers is a factor of key importance in the overall performance of devices based on oriented COF films. To identify potential COF candidates for optoelectronic applications, a detailed characterization of the physical and optoelectronic properties is highly desirable. Charge-carrier mobility of photoactive materials can be obtained from the electrical characteristics of a diode in which the photoactive material is sandwiched between two electrodes (Scheme 1). In the case of oriented thin COF films, this architecture allows for charge-carrier mobility directed exclusively along the stacked layers to be examined. We therefore initiated our study with the synthesis of oriented thin BDT-COF films on different glass-coated indiumdoped tin oxide electrodes (ITO). We followed our previously reported in situ film growth via the solvothermal cocondensation of benzo[1,2-b:4,5-b] dithiophene-2,6-diyldiboronic acid, BDTBA, and the polyol 2,3,6,7,10,11-hexahydroxytriphenylene, HHTP (Scheme 1). ${ }^{24}$ Briefly, stoichiometric amounts of BDTBA and HHTP monomers were placed in a stainless steel autoclave with an appropriate amount of mesitylene/dioxane $1: 1 \mathrm{v} / \mathrm{v}$ solvent mixture, which serves as the reaction medium. Later, a Teflon holder equipped with the desired conducting substrates was introduced into the autoclave. The autoclave was sealed and placed in a preheated oven at $100{ }^{\circ} \mathrm{C}$ for $12 \mathrm{~h}$. At the end of the solvothermal reaction, the substrates were recovered, washed with anhydrous acetone, and left overnight at $150{ }^{\circ} \mathrm{C}$ under vacuum. Along with the conductive substrates, a green powder was collected by vacuum filtration. The obtained powder was washed with anhydrous acetone to afford a highly crystalline COF powder, which is a first indication for the deposition of a crystalline film (Figure 1a).

A high-quality interface between the COF film and both electrodes is required to construct an efficient hole-only device. Top-view scanning electron microscope (SEM) analysis of the resulting electrodes reveals a smooth and homogeneous coverage (Figure S1a). Cross-section SEM images show a uniform dense thin film with a thickness of approximately 200 $\mathrm{nm}$ (Figure $1 \mathrm{~b}$ ). The surface morphology and roughness of the deposited films were additionally examined using atomic force microscopy (AFM). The AFM scan reveals a surface comprising intergrown domains forming a continuous, almost flawless film topography (Figure 1d,e). The average roughness (Ra), extracted from the film topography, was determined to be about $6 \mathrm{~nm}$, corresponding to a relatively smooth surface suitable for constructing a hole-only device. A detector scan Xray diffraction $(\mathrm{XRD})$ analysis indicates the preferential parallel orientation of the COF layers with respect to the electrode surface (Figure S1b). Grazing-incidence small-angle X-ray scattering analysis (GI-SAXS) confirmed that indeed preferential orientation of the COF layers was obtained, with the [001]-axis (c-axis) oriented orthogonal to the surface and rotational freedom around this axis, which is in agreement with our previous report (Figure 1c). ${ }^{24}$

The UV-vis absorption spectra of the BDT-COF films grown on quartz substrates appear as a superposition of the absorption spectra of the COF building blocks. This suggests limited in-plane electronic coupling between the COF building blocks, which are connected by the boronate ester linking groups. BDT-COF absorbs mainly in the UV region (Figure S2), corresponding to a direct optical band gap of $2.9 \mathrm{eV}$ (evaluated from a Tauc plot, Figure S3). The HOMO level of the BDT-COF was estimated from photoelectron spectroscopy in air (PESA) measurements (Figure S4) yielding a value of $-5.8 \pm 0.1 \mathrm{eV}$. The LUMO energy level can be evaluated by adding the optical band gap value to the HOMO energy level, $E_{\text {LUMO }}=-5.8+2.9=-2.9 \mathrm{eV}^{25,26}$

In our previous report, we observed charge transfer from an oriented BDT-COF thin film toward an electron-acceptor phase, resulting in the formation of hole-polarons within the framework. $^{24}$ Here, hole-only devices with the following architecture were prepared to study the columnar chargetransport properties of the BDT-COF: glass/ITO/MoO ${ }_{x} /$ $\mathrm{BDT}-\mathrm{COF} / \mathrm{MoO}_{x} / \mathrm{Au}$ (Scheme 1). ${ }^{27}$ The high work function of $\mathrm{MoO}_{x}$ permits the selective collection of holes from the devices. $^{28,29}$ For this purpose, a thin layer of $\mathrm{MoO}_{x}$ (about 10 $\mathrm{nm}$ ) was initially evaporated onto a glass-coated ITO electrode. Subsequently, an oriented BDT-COF thin film was grown as described above. To complete the device, $\mathrm{MoO}_{x}$ and gold were evaporated as top electrodes. At higher voltages, assuming ohmic injecting contacts and trap-free transport, the current 

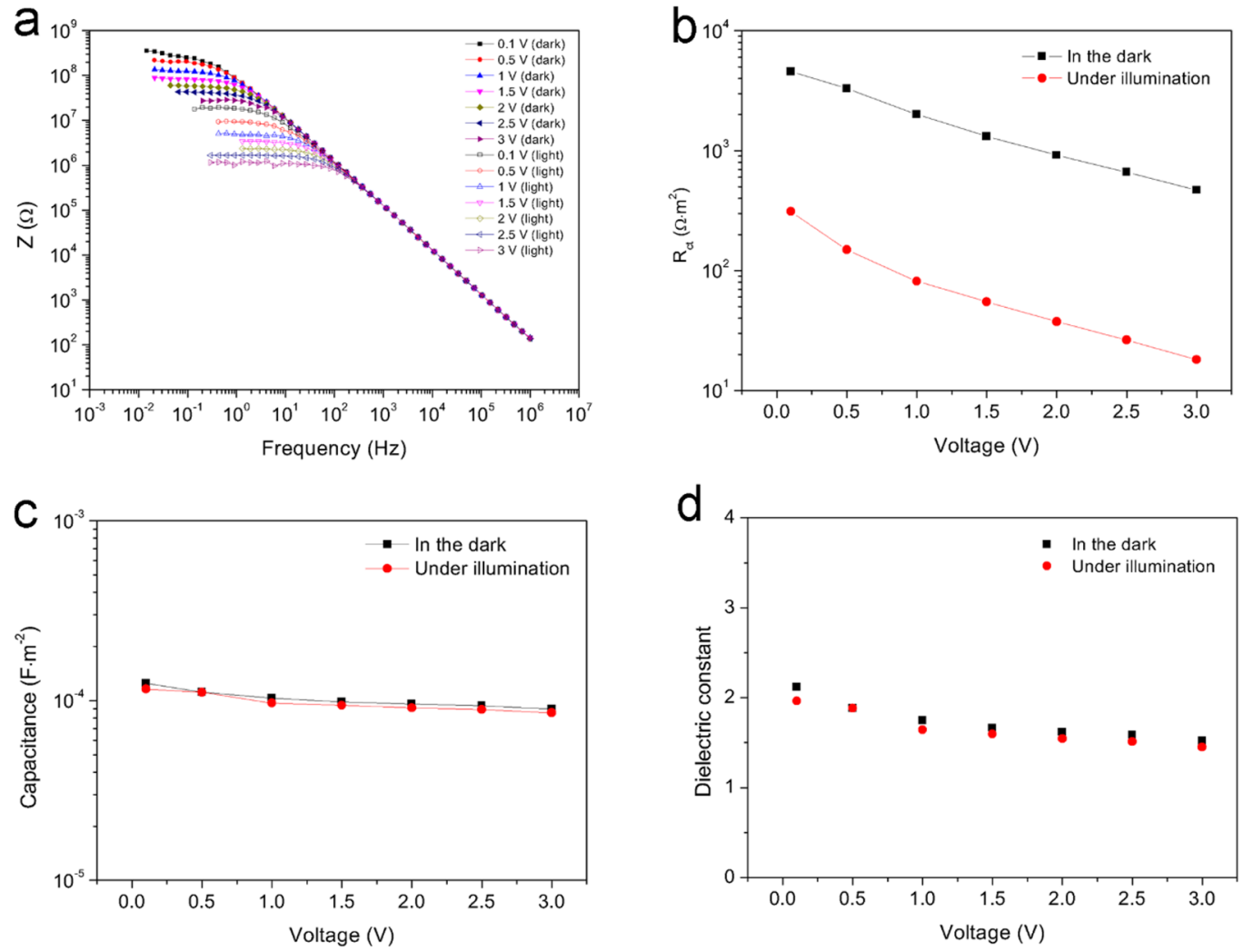

Figure 3. (a) Bode plot of impedance spectra of the HOD in the dark and under illumination. (b) Recombination resistance and (c) capacitance of the HOD in the dark and under illumination. (d) Dielectric constant for BDT-COF extracted from the impedance measurements.

measured is space-charge limited. ${ }^{30}$ The charge-carrier mobility can be estimated using the Mott-Gurney equation

$$
J=\frac{9}{8} \varepsilon_{0} \varepsilon_{\mathrm{r}} \mu \frac{V^{2}}{L^{3}}
$$

where $J$ is the current density, $\varepsilon_{\mathrm{r}}$ is the dielectric constant of the COF (we used 1.7 as derived from the impedance measurements), $\varepsilon_{0}$ is the permittivity of free space, $\mu$ is the charge carrier mobility, $L$ is the thickness of the active layer and $V$ is the voltage drop across the device.

To study the dependence of the charge-carrier mobility on the COF film thickness, HODs with BDT-COF film thickness ranging from 80 to $210 \mathrm{~nm}$ were fabricated (Figure S5). ${ }^{31}$ Thickness dependence measurements were performed in the dark, showing hole mobilities in the range of $3 \times 10^{-7}$ to $6 \times$ $10^{-9} \mathrm{~cm}^{2} \mathrm{~V}^{-1} \mathrm{~s}^{-1}$, where the highest values are obtained for the thinner COF films (Figure $2 \mathrm{~b}$ and Tables S1-S3). This indicates that the charge transport is limited in the bulk rather than by the COF/electrode interfaces. We attribute this thickness dependence to intrinsic electronic defects in the stacks of the BDT-COF layers. For a $90 \mathrm{~nm}$ thick film, 260 layers (calculated for $3.45 \AA$ interlayer distances obtained by the $\mathrm{X}$-ray diffraction data) must stack perfectly into a single $\mathrm{COF}$ domain to form a flawless stack between the two electrodes. In the case of an electronic defect in the stack, charges cannot efficiently evade the defect by traveling along alternative paths through the $a b$-plane, due to the electronic barriers imposed by the boronate ester linking groups.

Electrical conductivity measurements were carried out to evaluate the charge transport in the plane of the BDT-COF. Laterally placed interdigitated gold contacts were deposited on a glass substrate, on which the BDT-COF film was grown. Linear $J-V$ curves were obtained (Figure S7) in the measured voltage range. The measured conductivity in the plane was found to be in the range of $1-5 \times 10^{-7} \mathrm{~S} \mathrm{~cm}^{-1}$, and no significant light dependence was observed. In comparison, the electrical conductivity we report for the oriented BDT-COF films is higher than the two-point probe electrical conductivity value reported for randomly oriented BDT-COF crystallites in a compressed pellet. ${ }^{23}$ Interestingly, our values for the BDTCOF films are only slightly lower than those reported for an oriented imine-based COF film. ${ }^{19}$ This could be considered surprising since the conjugated imine bonds would be expected to significantly enhance charge transport in the plane. ${ }^{32}$ Nevertheless, the electrical conductivity is still relatively low compared to benchmark conductive polymers like P3HT and is expected to result in a high resistance for charge transport in the lateral direction.

Additionally, we performed light-dependent hole-mobility measurements using the hole-only devices comprising 200 nm BDT-COF films described above. In contrast to the conductivity measurements, a 3-fold increase in the average hole mobility (white light LED) was observed under illumination: $3 \times 10^{-8} \mathrm{~cm}^{2} \mathrm{~V}^{-1} \mathrm{~s}^{-1}$ (Table S4) under illumination compared to $5 \times 10^{-9} \mathrm{~cm}^{2} \mathrm{~V}^{-1} \mathrm{~s}^{-1}$ in the dark (Figure 2 and Table S3). This hints at the formation of photoinduced charges in the $\mathrm{COF}$, resulting in a charge density-dependent mobility in the film. ${ }^{33}$ We hypothesize that the increase in hole mobility under illumination results from the increased charge density, which allows electronic trap states to be filled. These electronic traps could originate from unreacted functional groups or defects in the COF structure. In 
a

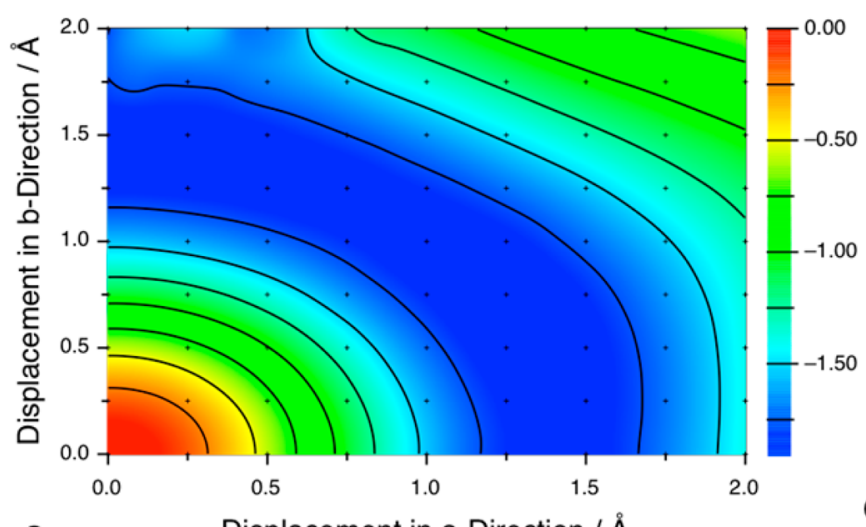

C

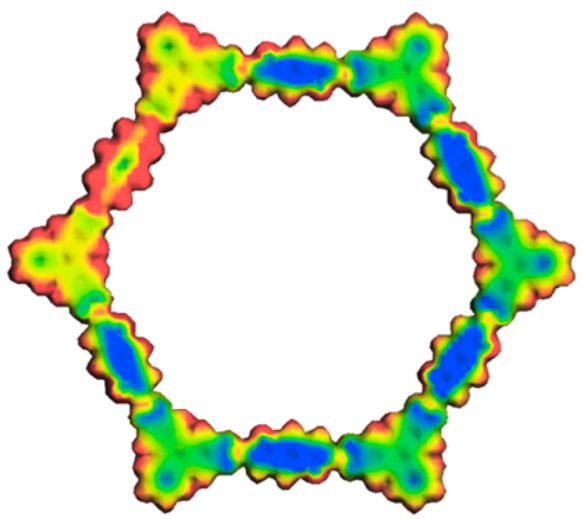

b

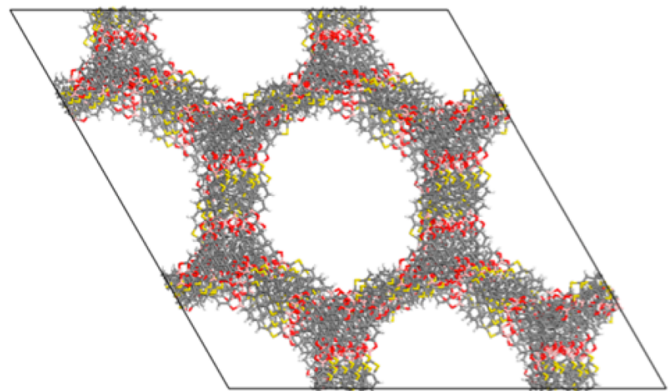

d

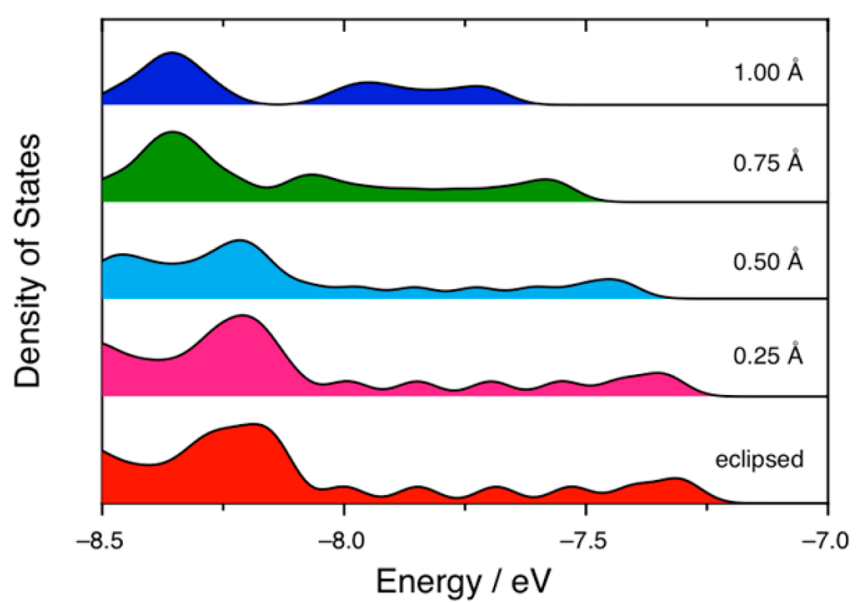

Figure 4. (a) Relative energy of BDT-COF structures with rigidly displaced layers (in eV). (b) Snapshot from a molecular dynamics simulation of a BDT-COF unit cell containing 20 layers. (c) AM1 molecular electrostatic potential plotted on the electron isodensity surface of a cationic BDT-COF ring in the gas phase (from - 0.1 (blue) to 0.3 (red) $\mathrm{Ha}^{-1}$ ). (d) AM1 density of states at the valence band edge of eclipsed and displaced BDT-COF structures.

particular, the presence of defects along the columnar stacks would be in good agreement with the above thicknessdependent mobility results. Despite the relatively low chargecarrier mobilities measured for the BDT-COF film compared to typical semiconducting polymers such as $\mathrm{P} 3 \mathrm{HT}$, the considerable increase in mobility under illumination indicates that the BDT-COF is indeed a photoactive material.

Impedance measurements performed with the BDT-COFbased HODs in the dark and under illumination further demonstrate the photoactive nature of the COF material (see Figure 3a). The values for the recombination resistance (Figure $3 \mathrm{~b})$ measured in the dark are an order of magnitude larger than those under illumination throughout the entire range of measurement conditions. The capacitance of the BDT-COFbased HODs remained constant for both dark and illuminated systems throughout the whole measurement. Furthermore, we estimated the dielectric constant, $\varepsilon_{\mathrm{r}}$, of the oriented BDT-COF films from the capacitance values using the following relationship

$$
\varepsilon_{\mathrm{r}}=\frac{C \times d}{\varepsilon_{0} \times A}
$$

where $C$ is the capacitance, $d$ is the thickness of the BDT-COF layer, $\varepsilon_{0}$ is the permittivity of free space, and $A$ is the area. Similar to the capacitance, the dielectric constant of the BDT$\mathrm{COF}$ is independent of the applied potential and has an average value of around 1.7. We attribute this low dielectric constant to the low density and highly porous structure of the COF film.

To gain further insights into the BDT-COF layer interactions and electronic structure, we turned to theoretical simulations. The experimental structural investigation of the BDT-COF points to an eclipsed COF layer geometry with open onedimensional channels. However, we expect that a direct spatial overlap of the polar $\mathrm{B}-\mathrm{O}$ bonds will lead to some degree of electrostatic repulsion. To quantify this effect, we performed density functional theory (DFT) calculations of different BDTCOF geometries, in which two rigid layers were displaced laterally relative to each other (see Figure $4 a$ ). The calculations were performed at the PBE+TS/DND level (see the Supporting Information for details). As expected, the potential energy hypersurface exhibits a maximum at the completely eclipsed geometry due to the electrostatic repulsion of partially charged atoms. A displacement of 1-2 $\AA$ in any direction leads to a significant stabilization, indicating that the structure will likely fluctuate between these low energy conformations at room temperature. These fluctuations were studied with classical molecular dynamics (MD) using the Dreiding force field (see the Supporting Information for details). From the isothermal-isobaric (NPT) ensemble MD simulations at ambient pressure and $300 \mathrm{~K}$, we obtain equilibrated lattice parameters that differ from the experimental values by only around $0.45 \%$. This small deviation confirms that our approach 
provides a good description of the system. As expected, the heteroatom-rich BDT-COF is strongly polarized, with $Q_{\mathrm{eq}}$ partial charges around 0.5 e for $B$ and -0.55 e for $O$. The structural strain associated with this electrostatic repulsion is relieved by horizontal movement of the planes relative to each other, in combination with a slight waving of the individual layers (Figure $4 b$ and Figure S8).

The evaluation of the BDT-COF electronic structure with respect to structural features can additionally assist in understanding the potential device performance. Primarily, we aimed at the localization of charge carriers within a COF layer. In Figure 4c, we show the electrostatic potential of a cation (hole) plotted on an electron-density isosurface $(\rho=0.03$ e $\AA^{-3}$ ) of an isolated COF ring in the gas-phase. Clearly, the charge carrier is localized on a single benzodithiophene unit. Hence, if a charge carrier cannot efficiently circumvent a defect in the BDT stack, for instance, through the COF layer, a decrease in charge-carrier collection at the respective electrode will occur.

Fully overlapped, eclipsed configuration oligothiophenes have been reported to exhibit the highest electronic coupling. ${ }^{34,35}$ One measure for electronic coupling in the condensed phase is the broadening of the valence band edge in the density of states (DOS), where high coupling leads to a stronger broadening. In Figure $4 d$, we show the DOS at the valence band edge for BDT-COF structures with different layer displacements. Evidently, the valence band edge is significantly less broadened in an $a b-1 \AA$ displaced geometry compared to the fully overlapped geometry. Therefore, in this particular COF system, the coupling is strongly reduced when the spatial overlap of the layers is small. This effect is unfavorable for charge-carrier transport, even in the absence of structural defects.

\section{CONCLUSIONS}

In summary, thin BDT-COF films were synthesized in an oriented fashion on different conductive substrates. Directional charge transport along the COF molecular columns and in the COF planes was studied. Columnar hole mobility was measured in a diode configuration by constructing hole-only devices (HODs). We showed that hole mobility along the BDT-COF stacks is feasible and that the charge transport depends on the COF film thickness. This dependency can be attributed to transport barriers within the BDT-COF stacks that cannot be easily overcome though alternative transport paths. Indeed, in-plane electrical conductivity measurements of oriented BDT-COF films indicated a relatively high resistivity toward charge migration attributed to the $\mathrm{COF}$ boronate ester linking groups. HODs measured under illumination show an enhanced hole mobility, thus demonstrating BDT-COF to be a photoactive material. Impedance measurements in the dark and under illumination further illustrate the photoactive nature of the BDT-COF; the recombination resistance decreases significantly under illumination. Moreover, we observed an exceptionally low dielectric constant for the BDT-COF films, which we attribute to the highly porous character of the COF. Simulations elucidate the relationship between the BDT-COF layer dynamics and the electronic structure. A certain degree of layer-to-layer displacement is expected for the BDT-COF due to the highly polarized $\mathrm{B}-\mathrm{O}$ bonds. This leads to a decrease of the layer-to-layer electronic coupling, translating into a narrowed density of states at the valence band edge. We therefore expect that thiophene COFs with maximized overlap between adjacent layers will show enhanced transport properties. Furthermore, providing alternative charge-migration paths within the COF planes will help to evade stacking defects and improve charge collection.

\section{METHODS}

General Methods. All materials (if not noted otherwise) were purchased from Aldrich, Fluka, or Acros in the common purities purum and puriss. 2,3,6,7,10,11-Hexahydroxytriphenylene (HHTP) was purchased from TCI Europe. All materials were used without further purification. The building block benzo[1,2-b:4,5- $\left.b^{\prime}\right]$ dithiophene-2,6-diyldiboronic acid (BDTBA) was prepared according to our previously reported synthesis route. Analytical data of intermediates were found to match literature data. ${ }^{20,36}$

BDT-COF Thin-Film Synthesis. A conventional stainless steel autoclave equipped with a $20 \mathrm{~mL}$ Teflon liner was charged with BDTBA (2) (16.2 mg, $0.058 \mathrm{mmol}$ ) and HHTP (12.5 mg, 0.038 $\mathrm{mmol}$ ). The reaction mixture was suspended in $5 \mathrm{~mL}$ of a $1: 1 \mathrm{v} / \mathrm{v}$ solution of mesitylene/dioxane. Two clean electrodes were placed horizontally on a homemade, $\prod$-shaped Teflon holder equipped with three slide compartments. The Teflon holder was placed into the starting material suspension in a way that the conductive side of the electrode faced the bottom of the autoclave. The autoclave was then heated for $12 \mathrm{~h}$ at $100{ }^{\circ} \mathrm{C}$. The substrates were then removed and washed with anhydrous acetone $(100 \mu \mathrm{L})$ and dried under a stream of $\mathrm{N}_{2}$. Thicker homogeneous COF films were obtained on the electrodes located in the lower Teflon holder compartment; thinner films were obtained on the electrode placed in the upper compartment.

Fabrication of Hole-Only Devices. Hole-only devices were prepared by adjusting published procedures. ${ }^{27,37-39}$ ITO substrates $(1.5 \times 2.0 \mathrm{~cm}$, VisionTek, $12-15 \Omega / \mathrm{sq})$ were etched using $\mathrm{Zn}$ powder and a $2 \mathrm{M} \mathrm{HCl}$ solution and cleaned with HELLMANEX soap and ethanol and were blown dry using pressured air. The substrates were plasma cleaned prior to the thermal deposition of the $\mathrm{MoO}_{x}$ layer (10 $\mathrm{nm})$ under vacuum $\left(1 \times 10^{-6} \mathrm{mbar}\right)$. The substrates were exposed to air and stored in a nitrogen-filled glovebox. Next, the BDT-COF layer was grown on the substrates as described above. A top electrode of $\mathrm{MoO}_{x}(10 \mathrm{~nm})$ followed by a layer of gold $(100 \mathrm{~nm})$ was deposited under vacuum $\left(1 \times 10^{-6} \mathrm{mbar}\right)$. The active area was $16 \mathrm{~mm}^{2}$. Current-voltage characteristics were recorded in air using a Metrohm potentiostat (PGSTAT302N) at a scan rate of $0.1 \mathrm{~V} \mathrm{~s}^{-1}$. Measurements under light were performed by illuminating the samples from the glass side, using a Metrohm cool white light LED (LDCCW, 6500 $\mathrm{K})$ with an intensity of approximately $1000 \mathrm{~W} \mathrm{~m}^{-2}$. The layer thickness was determined by using AFM measurements which were performed in tapping mode using a NanoInk DPN Stage microscope or with an SEM using a JEOL 6500F instrument.

In-Plane Conductivity Measurements. For conductivity measurements, precleaned glass substrates were prepared by deposition of $\mathrm{Ti} /$ $\mathrm{Au}(10 / 40 \mathrm{~nm}$ thick) interdigitating electrodes. The electrode pattern was designed for two point-probe measurements with a channel length of $250 \mu \mathrm{m}$, and a channel width of $0.2 \mathrm{~m}$ was used. BDT-COF films with a thickness of approximately $200 \mathrm{~nm}$ were grown as described above onto the interdigitated regions. $J-V$ curves were recorded under ambient conditions with a Keithley 2400 source meter at a scan rate of 0.1 and $1 \mathrm{~V} \mathrm{~s}^{-1}$, respectively. Measurements under light were performed by illuminating the samples from the glass side, using simulated AM 1.5G sunlight, with an incident power of $1000 \mathrm{~mW} \mathrm{~m}^{-2}$.

$\mathrm{X}$-ray diffraction analysis was carried out in reflection mode using a Bruker D8 Discover with Ni-filtered $\mathrm{Cu} \mathrm{K} \alpha$ radiation (1.5406 $\AA$ ) and a position-sensitive detector (LynxEye). Small-angle X-ray scattering (SAXS) was carried out with a SAXSpace instrument system (Anton Paar) equipped with a Princeton Instruments SCX CCD camera. The sample was subjected to the X-ray beam at a grazing angle of $0.24^{\circ}$ with respect to the primary beam. The X-rays scattered perpendicular $q(z)$ and parallel $q(y)$ to the surface normal were detected by the CDD camera covering wide angles, corresponding to $8.36^{\circ} 2 \theta$ in the $q(z)$ axis and $15.94^{\circ} 2 \theta$ in the $q(y)$ axis. The wavelength of the incident 
beam was $\lambda=0.154 \mathrm{~nm}(\mathrm{Cu} \mathrm{K} \alpha)$; the sample-detector distance was $168.15 \mathrm{~mm}$.

Scanning electron microscope images were recorded with JEOL $6500 \mathrm{~F}$ and Zeiss Ultra Plus field emission scanning electron microscopes. Samples were carbon coated prior to the measurements. The AFM data were collected with a nanoInk DPN-Stage atomic force microscope operated in tapping mode.

UV-vis spectra were recorded on a Lambda 1050 spectrometer (PerkinElmer) equipped with an integrating sphere. Thin films were measured in transmission geometry and corrected for reflection losses. The work function of the BDT-COF was measured using a Riken photoelectron spectrometer in air (PESA) (model AC-2) at $20 \mathrm{nW}$.

Impedance measurements were carried out using a potentiostat equipped with a frequency response analyzer (PGSTAT302N, Autolab, Metrohm) and LED driver. The measurements were carried out under cool white LED (LDCCW, Metrohm) irradiation at different applied DC voltages over the frequency range from $1 \mathrm{MHz}$ to $0.02 \mathrm{~Hz}$.

Computational Studies. The MD model system was based on the experimentally reported unit cell of BDT-COF, which we expanded to a hexagonal supercell approximately sized $74 \times 74 \times 75 \AA$ and containing 9577 atoms. The simulations were performed using the Dreiding force field as implemented in the Forcite Plus module of Materials Studio. Charges for the periodic COF network were calculated via the $Q_{\text {eq }}$ scheme. ${ }^{40}$ Electrostatic and van der Waals interactions were treated via the Ewald summation method with a $6 \AA$ cutoff on the repulsive contribution to the van der Waals energy.

The Dreiding force field has been shown to provide high quality descriptions of polythiophenes in the condensed phase when combined with charges from $\mathrm{ab}$ initio calculations or empirical schemes. ${ }^{41}$ We compared the charges calculated with the $Q_{\text {eq }}$ model to results from B3LYP/6-31G $(\mathrm{d}, \mathrm{p})$ calculations using the MK model. ${ }^{42-44}$ Both charges are in qualitative agreement, but the DFT calculations are artificially polarized due to the capping of the COF fragments used, while the $Q_{\mathrm{eq}}$ model could be applied directly to the periodic system.

Semiempirical molecular orbital theory calculations were performed with EMPIRE'13 using the AM1 Hamiltonian and periodic boundary conditions. ${ }^{45-48}$ DFT calculations were performed at the PBE/DND level using the TS dispersion correction and a $1 \times 1 \times 2 \mathrm{k}$ point mesh as implemented in DMol3. ${ }^{49-51}$

\section{ASSOCIATED CONTENT}

\section{S Supporting Information}

The Supporting Information is available free of charge on the ACS Publications website at DOI: 10.1021/acsnano.6b07692.

Materials and methods, BDT-COF HOD characterization and further physical description of oriented BDTCOF films, $\mathrm{MD}$ simulations (PDF)

\section{AUTHOR INFORMATION}

\section{Corresponding Authors}

*E-mail: dana.medina@cup.uni-muenchen.de.

*E-mail: bein@lmu.de.

\section{ORCID $\odot$}

Michiel L. Petrus: 0000-0001-7383-4952

Timothy Clark: 0000-0001-7931-4659

\section{Author Contributions}

${ }^{\perp}$ D.M., M.L.P., and A.N.J. contributed equally to this work.

\section{Notes}

The authors declare no competing financial interest.

\section{ACKNOWLEDGMENTS}

We gratefully acknowledge funding from the Bavarian Ministry of the Environment and Consumer Protection, the Bavarian
Network "Solar Technologies Go Hybrid", and the DFG Excellence Cluster Nanosystems Initiative Munich (NIM). M.L.P. acknowledges support from the German Federal Ministry of Education and Research (BMBF) under Agreement No. 01162525/1. A.N.J. acknowledges an Office of the Chief Executive Postdoctoral Fellowship (CSIRO Manufacturing). J.T.M. acknowledges support from the Alexander von Humboldt Foundation. The research leading to these results has received funding from the European Research Council under the European Union's Seventh Framework Programme (FP7/2007-2013)/ERC Grant Agreement No. 321339. We acknowledge Dr. Steffen Schmidt for the preparation of the SEM images.

\section{REFERENCES}

(1) Facchetti, A. pi-Conjugated Polymers for Organic Electronics and Photovoltaic Cell Applications. Chem. Mater. 2011, 23, 733-758.

(2) Mishra, A.; Bauerle, P. Small Molecule Organic Semiconductors on the Move: Promises for Future Solar Energy Technology. Angew. Chem., Int. Ed. 2012, 51, 2020-2067.

(3) Shaheen, S. E.; Brabec, C. J.; Sariciftci, N. S.; Padinger, F.; Fromherz, T.; Hummelen, J. C. 2.5\% Efficient Organic Plastic Solar Cells. Appl. Phys. Lett. 2001, 78, 841-843.

(4) Liao, H. C.; Ho, C. C.; Chang, C. Y.; Jao, M. H.; Darling, S. B.; $\mathrm{Su}, \mathrm{W}$. F. Additives for Morphology Control in High-Efficiency Organic Solar Cells. Mater. Today 2013, 16, 326-336.

(5) Liu, Y. S.; Chen, C. C.; Hong, Z. R.; Gao, J.; Yang, Y.; Zhou, H. P.; Dou, L. T.; Li, G.; Yang, Y. Solution-Processed Small-Molecule Solar Cells: Breaking the 10\% Power Conversion Efficiency. Sci. Rep. 2013, DOI: $10.1038 /$ srep03356.

(6) Poelking, C.; Tietze, M.; Elschner, C.; Olthof, S.; Hertel, D.; Baumeier, B.; Wurthner, F.; Meerholz, K.; Leo, K.; Andrienko, D. Impact of Mesoscale Order on Open-Circuit Voltage in Organic Solar cells. Nat. Mater. 2015, 14, 434-439.

(7) Liu, Y. H.; Zhao, J. B.; Li, Z. K.; Mu, C.; Ma, W.; Hu, H. W.; Jiang, K.; Lin, H. R.; Ade, H.; Yan, H. Aggregation and Morphology Control Enables Multiple Cases of High-Efficiency Polymer Solar Cells. Nat. Commun. 2014, 5, 5293.

(8) Cote, A. P.; Benin, A. I.; Ockwig, N. W.; O’Keeffe, M.; Matzger, A. J.; Yaghi, O. M. Porous, Crystalline, Covalent Organic Frameworks. Science 2005, 310, 1166-1170.

(9) Diaz, U.; Corma, A. Ordered Covalent Organic Frameworks, COFs and PAFs. From Preparation to Application. Coord. Chem. Rev. 2016, 311, 85-124.

(10) Feng, X.; Ding, X. S.; Jiang, D. L. Covalent Organic Frameworks. Chem. Soc. Rev. 2012, 41, 6010-6022.

(11) DeBlase, C. R.; Dichtel, W. R. Moving Beyond Boron: The Emergence of New Linkage Chemistries in Covalent Organic Frameworks. Macromolecules 2016, 49, 5297-5305.

(12) Ding, X.; Chen, L.; Honsho, Y.; Feng, X.; Saengsawang, O.; Guo, J.; Saeki, A.; Seki, S.; Irle, S.; Nagase, S.; Parasuk, V.; Jiang, D. An n-Channel Two-Dimensional Covalent Organic Framework. J. Am. Chem. Soc. 2011, 133, 14510-14513.

(13) Jin, S. B.; Furukawa, K.; Addicoat, M.; Chen, L.; Takahashi, S.; Irle, S.; Nakamura, T.; Jiang, D. L. Large Pore Donor-Acceptor Covalent Organic Frameworks. Chem. Sci. 2013, 4, 4505-4511.

(14) Jin, S. B.; Ding, X. S.; Feng, X.; Supur, M.; Furukawa, K.; Takahashi, S.; Addicoat, M.; El-Khouly, M. E.; Nakamura, T.; Irle.; et al. Charge Dynamics in A Donor-Acceptor Covalent Organic Framework with Periodically Ordered Bicontinuous Heterojunctions. Angew. Chem., Int. Ed. 2013, 52, 2017-2021.

(15) Dogru, M.; Bein, T. On the Road Towards Electroactive Covalent Organic Frameworks. Chem. Commun. 2014, 50, 5531-5546.

(16) Wan, S.; Gandara, F.; Asano, A.; Furukawa, H.; Saeki, A.; Dey, S. K.; Liao, L.; Ambrogio, M. W.; Botros, Y. Y.; Duan.; et al. Covalent Organic Frameworks with High Charge Carrier Mobility. Chem. Mater. 2011, 23, 4094-4097. 
(17) Ding, X.; Guo, J.; Feng, X.; Honsho, Y.; Guo, J.; Seki, S.; Maitarad, P.; Saeki, A.; Nagase, S.; Jiang, D. Synthesis of Metallophthalocyanine Covalent Organic Frameworks that Exhibit High Carrier Mobility and Photoconductivity. Angew. Chem., Int. Ed. 2011, 50, 1289-93.

(18) Medina, D. D.; Rotter, J. M.; Hu, Y. H.; Dogru, M.; Werner, V.; Auras, F.; Markiewicz, J. T.; Knochel, P.; Bein, T. Room Temperature Synthesis of Covalent-Organic Framework Films through VaporAssisted Conversion. J. Am. Chem. Soc. 2015, 137, 1016-1019.

(19) Cai, S. L.; Zhang, Y. B.; Pun, A. B.; He, B.; Yang, J. H.; Toma, F. M.; Sharp, I. D.; Yaghi, O. M.; Fan, J.; Zheng, S. R.; et al. Tunable Electrical Conductivity in Oriented Thin Films of TetrathiafulvaleneBased Covalent Organic Framework. Chem. Sci. 2014, 5, 4693-4700.

(20) Dogru, M.; Handloser, M.; Auras, F.; Kunz, T.; Medina, D.; Hartschuh, A.; Knochel, P.; Bein, T. A Photoconductive Thienothiophene-Based Covalent Organic Framework Showing Charge Transfer Towards Included Fullerene. Angew. Chem., Int. Ed. 2013, 52, 29202924

(21) Bertrand, G. H. V.; Michaelis, V. K.; Ong, T.-C.; Griffin, R. G.; Dinca, M. Thiophene-Based Covalent Organic Frameworks. Proc. Natl. Acad. Sci. U. S. A. 2013, 110, 4923-8.

(22) Lohse, M. S.; Rotter, J. M.; Margraf, J. T.; Werner, V.; Becker, M.; Herbert, S.; Knochel, P.; Clark, T.; Bein, T.; Medina, D. D. From Benzodithiophene to Diethoxy-Benzodithiophene Covalent Organic Frameworks - Structural Investigations. CrystEngComm 2016, 18, 4295-4302.

(23) Duhovic, S.; Dinca, M. Synthesis and Electrical Properties of Covalent Organic Frameworks with Heavy Chalcogens. Chem. Mater. 2015, 27, 5487-5490.

(24) Medina, D. D.; Werner, V.; Auras, F.; Tautz, R.; Dogru, M.; Schuster, J.; Linke, S.; Doblinger, M.; Feldmann, J.; Knochel, P.; et al. Oriented Thin Films of a Benzodithiophene Covalent Organic Framework. ACS Nano 2014, 8, 4042-4052.

(25) Davis, R. J.; Lloyd, M. T.; Ferreira, S. R.; Bruzek, M. J.; Watkins, S. E.; Lindell, L.; Sehati, P.; Fahlman, M.; Anthony, J. E.; Hsu, J. W. P. Determination of Energy Level Alignment at Interfaces of Hybrid and Organic Solar Cells Under Ambient Environment. J. Mater. Chem. 2011, 21, 1721-1729.

(26) Winzenberg, K. N.; Kemppinen, P.; Fanchini, G.; Bown, M.; Collis, G. E.; Forsyth, C. M.; Hegedus, K.; Singh, T. B.; Watkins, S. E. Dibenzo [b,def] chrysene Derivatives: Solution-Processable Small Molecules that Deliver High Power-Conversion Efficiencies in Bulk Heterojunction Solar Cells. Chem. Mater. 2009, 21, 5701-5703.

(27) Petrus, M. L.; Morgenstern, F. S. F.; Sadhanala, A.; Friend, R H.; Greenham, N. C.; Dingemans, T. J. Device Performance of SmallMolecule Azomethine-Based Bulk Heterojunction Solar Cells. Chem. Mater. 2015, 27, 2990-2997.

(28) Shim, J. W.; Fuentes-Hernandez, C.; Zhou, Y. H.; Dindar, A.; Khan, T. M.; Giordano, A. J.; Cheun, H.; Yun, M.; Marder, S. R.; Kippelen, B. Inverted Tandem Polymer Solar Cells with Polyethylenimine-Modified $\mathrm{MoO}_{\mathrm{X}} / \mathrm{Al}_{2} \mathrm{O}_{3}: \mathrm{ZnO}$ Nanolaminate as the Charge Recombination Layers. Adv. Energy Mater. 2014, 4, 1400048.

(29) Battaglia, C.; Yin, X. T.; Zheng, M.; Sharp, I. D.; Chen, T.; McDonnell, S.; Azcatl, A.; Carraro, C.; Ma, B. W.; Maboudian, R.; et al. Hole Selective $\mathrm{MoO}_{\mathrm{x}}$ Contact for Silicon Solar Cells. Nano Lett. 2014, 14, 967-971.

(30) Chuang, S.; Battaglia, C.; Azcatl, A.; McDonnell, S.; Kang, J. S.; Yin, X. T.; Tosun, M.; Kapadia, R.; Fang, H.; Wallace, R. M.; et al. $\mathrm{MoS}_{2}$ P-type Transistors and Diodes Enabled by High Work Function $\mathrm{MoO}_{\mathrm{x}}$ Contacts. Nano Lett. 2014, 14, 1337-1342.

(31) Blom, P. W. M.; Tanase, C.; de Leeuw, D. M.; Coehoorn, R. Thickness Scaling of the Space-Charge-Limited Current in Poly(pPhenylene Vinylene). Appl. Phys. Lett. 2005, 86, 92105.

(32) Koole, M.; Frisenda, R.; Petrus, M. L.; Perrin, M. L.; van der Zant, H. S. J.; Dingemans, T. J. Charge Transport Through Conjugated Azomethine-Based Single Molecules for Optoelectronic Applications. Org. Electron. 2016, 34, 38-41.

(33) Snaith, H. J.; Gratzel, M. Light-Enhanced Charge Mobility in a Molecular Hole Transporter. Phys. Rev. Lett. 2007, 98, 177402.
(34) Liu, H. G.; Bremond, E.; Prlj, A.; Gonthier, J. F.; Corminboeuf, C. Adjusting the Local Arrangement of pi-Stacked Oligothiophenes through Hydrogen Bonds: A Viable Route to Promote Charge Transfer. J. Phys. Chem. Lett. 2014, 5, 2320-2324.

(35) Kubas, A.; Hoffmann, F.; Heck, A.; Oberhofer, H.; Elstner, M.; Blumberger, J. Electronic Couplings for Molecular Charge Transfer: Benchmarking CDFT, FODFT, and FODFTB Against High-Level ab initio Calculations. J. Chem. Phys. 2014, 140, 104105.

(36) Kashiki, T.; Shinamura, S.; Kohara, M.; Miyazaki, E.; Takimiya, K.; Ikeda, M.; Kuwabara, H. One-pot Synthesis of Benzo [b] Thiophenes and Benzo [b] Selenophenes From o-Halo-Substituted Ethynylbenzenes: Convenient Approach to Mono-, Bis-, and TrisChalcogenophene-Annulated Benzenes. Org. Lett. 2009, 11, 24732475.

(37) Matsushima, T.; Kinoshita, Y.; Murata, H. Formation of Ohmic Hole Injection by Inserting an Ultrathin Layer of Molydenum Trioxide Between Indium Tin Oxide and Organic Hole-Transporting Layers. Appl. Phys. Lett. 2007, 91, 253504.

(38) Nicolai, H. T.; Wetzelaer, G. A. H.; Kuik, M.; Kronemeijer, A. J.; de Boer, B.; Blom, P. W. M. Space-Charge-Limited Hole Current in Poly(9,9-dioctylfluorene) Diodes. Appl. Phys. Lett. 2010, 96, 172107.

(39) Petrus, M. L.; Bouwer, R. K. M.; Lafont, U.; Murthy, D. K. H.; Kist, R. J. P.; Böhm, M. L.; Olivier, Y.; Savenije, T. J.; Siebbeles, L. D. A.; Greenham, N. C.; et al. Conjugated Poly(azomethine)s via Simple One-step Polycondensation Chemistry: Synthesis, Thermal and Optoelectronic Properties. Polym. Chem. 2013, 4, 4182-4191.

(40) Rappe, A. K.; Goddard, W. A. Charge Equilibration for Molecular Dynamics Simulations. J. Phys. Chem. 1991, 95, 3358-3363.

(41) Alexiadis, O.; Mavrantzas, V. G. All-Atom Molecular Dynamics Simulation of Temperature Effects on the Structural, Thermodynamic, and Packing Properties of the Pure Amorphous and Pure Crystalline Phases of Regioregular P3HT. Macromolecules 2013, 46, 2450-2467.

(42) Becke, A. D. Density-Functional Thermochemistry. III. The Role of Exact Exchange. J. Chem. Phys. 1993, 98, 5648-5652.

(43) Francl, M. M.; Pietro, W. J.; Hehre, W. J.; Binkley, J. S.; Gordon, M. S.; Defrees, D. J.; Pople, J. A. Self-Consistent molecular orbital methods. XXIII. A polarization-type basis set for second-row elements. J. Chem. Phys. 1982, 77, 3654-3665.

(44) Singh, U. C.; Kollman, P. A. An Approch To Computing Electrostatic Charges for Molecules. J. Comput. Chem. 1984, 5, 129145

(45) Hennemann, M.; Clark, T. EMPIRE: a Highly Parallel Semiempirical Molecular Orbital Program: 1: Self-Consistent Field Calculations. J. Mol. Model. 2014, 20, 2331.

(46) Margraf, J. T.; Hennemann, M.; Meyer, B.; Clark, T. EMPIRE: a Highly Parallel Semiempirical Molecular Orbital Program: 2: Periodic Boundary Conditions. J. Mol. Model. 2015, 21, 144.

(47) Dewar, M. J. S.; Zoebisch, E. G.; Healy, E. F.; Stewart, J. J. P. The Development and use of Quantum-Mechanical MolecularModels. 76. AM1: New General Purpose Quantum-Mechanical Molecular Model. J. Am. Chem. Soc. 1985, 107, 3902-3909.

(48) Perdew, J. P.; Burke, K.; Ernzerhof, M. Generalized Gradient Approximation Made Simple. Phys. Rev. Lett. 1997, 78, 1396-1396.

(49) Tkatchenko, A.; Scheffler, M. Accurate Molecular Van Der Waals Interactions from Ground-State Electron Density and FreeAtom Reference Data. Phys. Rev. Lett. 2009, 102, 73005.

(50) Monkhorst, H. J.; Pack, J. D. Special Points for Brillouin-Zone Integrations. Phys. Rev. B 1976, 13, 5188-5192.

(51) Delley, B. An All-Electron Numerical-Method for Solving the Local Density Functional for Polyatomic Molecules. J. Chem. Phys. 1990, 92, 508-517. 